\title{
Correction to: Peripheral markers of autophagy in polyglutamine diseases
}

Giorgia Puorro $^{1}$ - Angela Marsili ${ }^{1} \cdot$ Francesca Sapone $^{1} \cdot$ Chiara Pane $^{1} \cdot$ Anna De Rosa ${ }^{1}$. Silvio Peluso ${ }^{1}$ - Giuseppe De Michele ${ }^{1}$ - Alessandro Filla ${ }^{1} \cdot$ Francesco Saccà $^{1}$

Published online: 17 November 2017

(C) Springer-Verlag Italia S.r.1., part of Springer Nature 2017

Correction to: Neurol Sci (2017)

https://doi.org/10.1007/s10072-017-3156-6

Dr. Peluso's given name and family name were initially interchanged inadvertently. The correct names have been corrected above. The original article was corrected.

Francesco Saccà

francesco.sacca@unina.it

1 Department of Neurosciences, Odontostomatological and

Reproductive Sciences, University Federico II, Via Pansini, 5,

80131 Naples, NA, Italy 